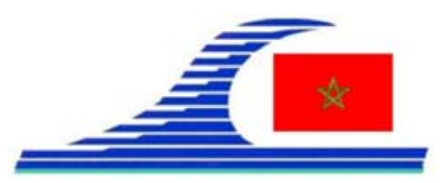

Conférence Méditerranéenne Côtière et Maritime

EDITION 2, TANGER, MAROC (2011)

Coastal and Maritime Mediterranean Conference

Disponible en ligne - http://www.paralia.fr - Available online

\title{
Les géosciences marines : un outil stratégique non exploité dans les politiques de développement durable au Maroc
}

\author{
Naima HAMOUMI ${ }^{1}$ \\ 1. Laboratoire Océanologie et Géodynamique des Bassins sédimentaires, Faculté des \\ Sciences, Université Mohammed V - Agdal, Rabat, Maroc. \\ naimahamoumi@yahoo.fr
}

\section{Résumé :}

Malgré une vocation maritime favorisée par un contexte géographique exceptionnel lui permettant d'avoir 3 façades (totalisant une longueur de $3500 \mathrm{~km}$ ) sur trois types de domaines marins (mer Méditerranéenne, détroit de Gibraltar et océan Atlantique), le Maroc souffre d'un retard considérable en géosciences marines du fait l'inexistence d'une politique efficiente dans ce domaine. Ainsi la plupart des écosystèmes littoraux et marins, qui représentent une ressource inestimable et contribuent largement à l'économie nationale, sont dans un état de dégradation avancé. De plus, en dehors de-la plateforme du détroit de Gibraltar qui a été bien étudiée dans le cadre du Projet Liaison Fixe, dans les autres domaines littoraux et marins, il y a en général une méconnaissance des modèles de circulation, du fonctionnement et des caractéristiques géologiques du substratum.

Ce travail se veut donc un plaidoyer pour la sensibilisation des décideurs à l'intérêt et l'urgence de mettre en œuvre une stratégie cohérente et pertinente pour développer la recherche et la formation en géosciences marines afin de faire face aux :

- risques naturels et anthropiques,

- enjeux d'ordre scientifique, socioéconomique et environnemental,

- défis qui se posent au Maroc dans le cadre du choix stratégique d'une politique de développement économique et humain, des fondements de la nouvelle constitution, de la régionalisation, de la mondialisation, du changement climatique, de la croissance démographique, des objectifs du millénaire et des engagements internationaux.

\section{Mots-clés :}

Géosciences marines - Développement durable - Formation - Recherche - Maroc

\section{Introduction}

Le Maroc a une situation géographique privilégiée qui lui permet d'avoir des façades (totalisant une longueur de $3500 \mathrm{~km}$ ) sur trois types de domaines marins (mer Méditerranéenne, détroit de Gibraltar et océan Atlantique) et une zone économique exclusive (ZEE) d'une superficie de 1 million de $\mathrm{km}^{2}$. En plus de leur intérêt scientifique et écologique, ces domaines marins et littoraux (dont de nombreux 
La connaissance de la Mer :

un vecteur du développement durable en Méditerranée

écosystèmes sont classés "SIBE"), jouent un rôle important dans l'économie du pays (pêche, aquaculture, tourisme), appelé à se renforcer dans le futur. Cependant, en dépit des enjeux et des défis qui se posent, le Maroc souffre d'un retard considérable dans le domaine des géosciences marines. Ce travail se veut un plaidoyer pour la sensibilisation des décideurs à l'intérêt et l'urgence d'adopter une stratégie nationale efficiente pour la promotion et le développement des géosciences marines au Maroc.

\section{Démarche}

Ce travail est basé sur une analyse du contexte national et du "Système National de Recherche et de l'Innovation" (SNRI), une évaluation de l'état d'avancement des géosciences marines dans les universités marocaines (moyens humains et matériels, structures de recherche, programmes de recherche, production scientifiques, offres de formation) et une revue de l'état des connaissances et de l'environnement des domaines littoraux et marins marocains. A la lumière de ce diagnostic et des enjeux et défis qui se posent, il propose les grandes lignes d'une stratégie nationale pour la promotion des géosciences marines.

\section{Le constat}

\subsection{Les contraintes et les dysfonctionnements du SNRI}

La prise de conscience sur l'intérêt et le rôle de la recherche scientifique dans le développement socio-économique qui s’est concrétisée par les réformes initiées en 1997 et par les différentes stratégies qui ont accompagné la loi 01-00 (BO n 4800 du 1/06/2000), a certes permis de réaliser quelques avancées. Cependant, ces stratégies n’ont pas réussi à ancrer des mécanismes et des procédures efficients pour une véritable politique de recherche et de formation cohérente et intégrée. De plus, les géosciences marines qui constituent pourtant un outil stratégique incontournable pour la connaissance et la gestion des espaces maritimes, ont été complètement négligées. Et de ce fait, les contraintes et les difficultés qui représentaient déjà un frein à leur développement, ont été amplifiées.

La balkanisation dont souffrait déjà la communauté des géologues marins qui ne représentent que 3,8\% du potentiel scientifique national, a été aggravée par l'approche utilisée pour l'accréditation des laboratoires de recherche dans le cadre du programme national de la restructuration de la recherche universitaire lancé par le Ministère de tutelle en 2004. En effet, au lieu de fédérer les moyens humains selon des critères scientifiques et objectifs, la restructuration a tout simplement rayé de la carte de l'université des laboratoires qui ont fait leur preuve et contribué au rayonnement scientifique du pays. Cette communauté va se réduire encore plus avec les départs à la retraite imminents dans le courant des 10 prochaines années. Ainsi, les travaux axés le plus souvent sur le littoral, sont menés en général dans une logique individuelle ou à 
l'échelle de petites équipes. De plus, du fait de l'absence de coordination et de planification à l'échelle nationale, les études sont souvent répétitives et dans la plupart des cas les travaux existants ne sont jamais capitalisés.

Le secteur universitaire souffre également de l'inexistence d'équipements lourds pour les investigations en haute mer et dans le littoral. De plus, les moyens nécessaires au fonctionnement et à la valorisation de la recherche restent dérisoires sinon inexistants pour les équipes qui n’ont pas été accréditées lors de la restructuration. Enfin, les marocains ne sont impliqués dans les campagnes internationales qui ont lieu dans la zone économique exclusive (ZEE) que rarement et en tant qu'observateur.

Par ailleurs, le nombre de doctorants en chute libre depuis quelques années, du fait des difficultés matérielles précitées, s'est réduit de manière dramatique avec les dernières vagues d'embauche. Le manque d'engouement à préparer des thèses est lié à la rareté et au taux des bourses de soutien, la quasi absence de recrutement dans les universités et l'accès à l'échelle 11 avec le diplôme du DESA et du Master. Les formations en géosciences marines, qui bien que rares et ponctuelles, ont été organisées dans le cadre du DESA et du DESS, sont devenues quasi inexistantes avec l'avènement du système LMD, l'enseignement des géosciences marines ne se fait plus que dans le cadre d'un module de filière, du parcours d'une licence ou d'une option dans un Master.

\subsection{Etat des connaissances sur l'environnement des domaines littoraux et marins}

Dans les zones littorales, malgré les nombreuses études réalisées, l’hydrologie et les trajectoires des transits sédimentaires sont souvent encore méconnues. Par ailleurs, la plupart des écosystèmes sont déjà dans un état de dégradation avancé (déséquilibres sédimentaires, pollution importante). En dehors de la plateforme du Détroit de Gibraltar qui a été bien étudiée dans le cadre du Projet Liaison Fixe, dans les autres domaines littoraux et marins, il est aujourd'hui difficile de connaître avec précision les modes de circulation, la topographie détaillée, la lithologie et la structuration du substratum ainsi que les ressources non vivantes qui lui sont associées (HAMOUMI, 2008).

\section{Les enjeux et les défis : recommandations}

Les enjeux sont à la fois d'ordre scientifique, socioéconomique et environnemental. Par ailleurs, les domaines littoraux et marins marocains sont menacés d'une part, par les risques sismiques et les tsunamis du fait de leur situation géographique et de leur contexte géotectonique et d'autre part, par une élévation du niveau de la mer en relation avec le réchauffement climatique. De plus, ils constituent des zones à haut risque pour les pollutions par les rejets opérationnels et les marées noires (HAMOUMI, 2008). Il n'est donc plus permis de continuer à tourner le dos à la mer et à ignorer le rôle sociétal des géosciences marines pour une gestion optimale du présent et du futur. Les défis qui se posent au Maroc dans le cadre du choix stratégique d'une politique de développement économique et humain, des fondements de la nouvelle constitution, de 
La connaissance de la Mer :

un vecteur du développement durable en Méditerranée

la régionalisation, de la mondialisation, du changement climatique, de la croissance démographique, des objectifs du millénaire et des engagements internationaux, appellent :

i) au renforcement des capacités pour favoriser l'émergence d'une économie du savoir avec la création de nouveaux métiers de la mer,

ii) à la promotion d'une économie de la mer dans le cadre d'un développement durable,

iii) à la nécessité absolue d’une gestion intégrée pour la préservation et la valorisation des écosystèmes littoraux qui sont des milieux fragiles, de plus en plus convoités et mal réglementés.

La question de la promotion des géosciences marines se pose donc avec acuité et l'université est appelée à y jouer un rôle essentiel, conformément à sa mission. Par conséquent il est vivement recommandé d'adopter une stratégie qui devrait cibler en plus de l'instauration d'une culture environnementale et de mesures de contrôle et de réglementation, en particulier pour les études de faisabilité et d’impact, la mise en place de mécanismes et d'outils efficients pour la coordination et la planification de la recherche, le renforcement des moyen humains, la mobilisation de moyens pour l'acquisition des équipements scientifiques nécessaires et pour le fonctionnement et la valorisation de la recherche, le renforcement de la coopération et enfin, la mise en place de formations adéquates au niveau de la licence (HAMOUMI, 2010) du Master et des écoles doctorales.

\section{Conclusions}

A l'issue de ce diagnostic, il apparaît clairement que le Maroc ne peut pas se permettre de continuer à faire l'économie de cet outil stratégique que sont les géosciences marines. Les risques et les nombreux enjeux et défis qui se posent, appellent à l'intérêt et l'urgence de mettre en œuvre une stratégie cohérente et pertinente pour le développement des géosciences marines afin de réussir la préservation et la valorisation espaces littoraux et marins qui jouent un rôle primordial dans l'économie du pays.

\section{Références bibliographiques}

HAMOUMI N. (2008). Les sciences de la mer: un outil stratégique pour le développement durable au Maroc., Revue prospectives universitaires, Université Mohammed V - Agdal, n 1, pp 199-219.

HAMOUMI N. (2010). Pour une efficience de la reforme dans les filières scientifiques et une meilleure articulation entre les filières en sciences de la terre et l'environnement socio-économique. Revue prospectives universitaires, Université Mohammed V Agdal, n 2, pp 17-43. 